Yoshiyuki Kitaoka

Nagoya Math. J.

Vol. 42 (1971), 89-93

\title{
ON A SPACE OF SOME THETA FUNGTIONS
}

\author{
YOSHIYUKI KITAOKA
}

In the theory of modular forms there is an interesting problem whether every modular form can be expressed as a linear comination of theta functions. For this Eichler proved in [1] that for a sufficiently large prime $q$ all modular forms of degree $-2 m(m=1,2, \cdots)$ for $\Gamma_{0}(q)$ can be represented by linear combinations of theta functios of degree $-2 m$ with level 1 and $q$. We prove this theorem for $q=2,3,5$ and 11 by using a theorem of Siegel for $q=2,3,5$ and a general result of Eichler for $q=11$. The former method is shown in Schoeneberg [2].

Before our statement, it should be recalled: for an even positive $4 m \times 4 m$ matrix $Q$ with level $N$ and square discriminant, the theta function

$$
\vartheta(\tau, Q)=\sum_{\xi \in Z^{4 m}} e^{\pi i t \xi Q \xi \pi}
$$

is a modular form of degree $-2 m$ for $\Gamma_{0}(N)$, i.e. of type $(-2 m, N, 1)$ in the sense of Hecke.

Theorem. For $q=2,3,5$ and 11 all modular forms of degree $-2 m(m=1,2$, -.) for $\Gamma_{0}(q)$ can be represented by linear combinations of theta functions of type $(-2 m, q, 1)$ and $(-2 m, 1,1)$.

Proof for $q=2$. Let $d_{m}$ (resp. $e_{m}$ ) be the dimension of the space $\mathfrak{M}(m)$ (resp. $\mathfrak{S}(m)$ ) of modular forms (resp. cusp forms) of degree $-2 m$ for $\Gamma_{0}(2)$. Then it is well known that

$$
\left\{\begin{array}{lll}
d_{m}=\left[\frac{m}{2}\right]+1 & \text { for } & m \geq 1, \\
e_{m}=0 & \text { for } & m=1, \\
e_{m}=\left[\frac{m}{2}\right]-1 & \text { for } & m \geq 2 .
\end{array}\right.
$$

Let $A$ be an even positive $4 \times 4$ matrix with level 2 and determinant 4 , for

Received May 4, 1970. 
example

$$
A=\left(\begin{array}{llll}
2 & 1 & 1 & 1 \\
1 & 2 & 0 & 0 \\
1 & 0 & 2 & 0 \\
1 & 0 & 0 & 2
\end{array}\right)
$$

and $M$ be an eveen positve $8 \times 8$ matrix with determinat 1 , for example

$$
M=\left(\begin{array}{rrrrrrrr}
2 & 1 & & & & & & \\
1 & 2 & 1 & & & & & \\
& 1 & 4 & 3 & & & & \\
& & 3 & 4 & 5 & & & \\
& & & 5 & 20 & 3 & & \\
& & & & 3 & 12 & 1 & \\
& & & & & 1 & 4 & 1 \\
& & & & & & 1 & 2
\end{array}\right)
$$

by Minkowski. Then $\vartheta(\tau, A)$ is a modular form of degree -2 for $\Gamma_{0}(2)$ and $\vartheta(\tau, M)$ is a modular form of degree -4 for $\Gamma(1)$. There are two inequivalent cusps 0 and $\infty$ for $\Gamma_{0}(2)$, and

$$
\begin{aligned}
& \vartheta(\tau, A)=1 \quad \text { at } \quad \tau=\infty, \vartheta(\tau, M)=1 \quad \text { at } \quad \tau=\infty, \\
& \vartheta(\tau, A)=-\frac{1}{2} \quad \text { at } \quad \tau=0, \vartheta(\tau, M)=1 \quad \text { at } \quad \tau=0 .
\end{aligned}
$$

Under these preparations we prove the theorem inductively. Firstly it is clear by the dimension formula (1) that $\mathfrak{M}(1)=C\{\vartheta(\tau, A)\}$,

$$
\mathfrak{M}(2)=\boldsymbol{C}\left\{\vartheta(\tau, A)^{2}, \vartheta(\tau, M)\right\} \text { and } \mathfrak{M}(3)=\boldsymbol{C}\left\{\vartheta(\tau, A)^{3}, \vartheta(\tau, A) \vartheta(\tau, M)\right\} .
$$

Secondly we prove the theorem for $\mathfrak{M}(4)$ by using Siegel's theorem. Put

$$
B=\left(\begin{array}{cccc}
A & & & \\
& A & & \\
& & A & \\
& & & A
\end{array}\right)
$$

Then $B$ is an even positive $16 \times 16$ matrix with level 2 and determinant $4^{4}$ and owing to Siegel [3] 


$$
F(\tau, B)=\frac{1}{M(B)} \sum_{B_{k}} \frac{\vartheta\left(\tau, B_{k}\right)}{E_{k}}
$$

can be represented by Eisenstein series with level 2, where $B_{k}$ runs over all representatives fo the classes in the genus of $B, E_{k}$ is the ordre of the unit group of $B_{k}$ and $M(B)=\sum \frac{1}{E_{k}}$. Since $F(\tau, B)=1$ at $\tau=\infty$ and $F(\tau, B)=$ $\frac{1}{16}$ at $\tau=0$

$$
\begin{aligned}
F(\tau, B) & =\frac{480}{17}\left(G_{8}(\tau)-G_{8}(2 \tau)\right)+480 G_{8}(2 \tau) \\
& =1+\frac{480}{17} e^{2 \pi i \tau}+\cdots \cdots,
\end{aligned}
$$

where $G_{l}(\tau)$ is an Eisenstein series with level 1 defined by

$$
\begin{aligned}
G_{l}(\tau) & =\frac{(l-1) !(-1)^{\frac{l}{2}}}{2(2 \pi)^{l}} \sum_{c, d \in Z}^{\prime} \frac{1}{(c \tau+d)^{l}} \\
& =\frac{(l-1) !(-1)^{-\frac{l}{2}}}{(2 \pi)^{l}} \zeta(l)+\sum_{n=1}^{\infty}\left(\sum_{d \mid n} d^{l-1}\right) e^{2 \pi i n \tau} .
\end{aligned}
$$

Now $\frac{480}{17}$ is not an integer. Hence among the above theta functinos $\vartheta\left(\tau, B_{k}\right)$ we can take some $\vartheta\left(\tau, B_{k_{0}}\right)$, linealy independent to $\vartheta(\tau, B)$. Consequently $\mathfrak{M}(4)$ is $\boldsymbol{C}\left\{\vartheta(\tau, M)^{2}, \vartheta(\tau, B), \vartheta\left(\tau, B_{k_{0}}\right)\right\}$, since $\vartheta(\tau, B)=\vartheta\left(\tau, B_{k_{0}}\right)=1$ at $\tau=\infty$ and $\vartheta(\tau, B)=\vartheta\left(\tau, B_{k_{0}}\right)=\frac{1}{16}$ at $\tau=0$, and so $\vartheta(\tau, M)^{2} \notin C\left\{\vartheta(\tau, B), \vartheta\left(\tau, B_{k_{0}}\right)\right\}$. Lastly since $\mathfrak{M}(m)=\mathfrak{M}(m-1) \times \vartheta(\tau, A)$ for any odd integer $m \geq 3$, we asusme that $m$ is even. For $m \geq 6, e_{m}=d_{m-4}$. Therefore $\subseteq(m)$ is the product of $\mathfrak{M}(m-4)$ and a one-dimensional space spanned by a cusp form of degree -8. Moreover, since $\vartheta(\tau, A)^{m}=\vartheta(\tau, M)^{\frac{m}{2}}=1$ at $\tau=\infty, \vartheta(\tau, A)^{m}=2^{-m}$ at $\tau=0$, and $\vartheta(\tau, M)^{\frac{m}{2}}=1$ at $\tau=0$, we can deduce that $\mathfrak{M}(m)$ is generated by $\vartheta(\tau, A)^{m}, \vartheta(\tau, M)^{\frac{m}{2}}$ and cusp forms in $\Im(m)$. Thus we have completed the proof for $q=2$.

For $q=3,5$ and 11 the proof is analogous under some modifications and we simply point out them.

The dimersion formula (1) should be replaced by the followings: 


$$
\left\{\begin{array} { l } 
{ d _ { s } = [ \frac { 2 } { 3 } s ] + 1 } \\
{ e _ { 1 } = 0 } \\
{ e _ { t } = [ \frac { 2 } { 3 } t ] - 1 , }
\end{array} \quad \left\{\begin{array} { l } 
{ d _ { s } = 2 [ \frac { s } { 2 } ] + 1 } \\
{ e _ { 1 } = 0 } \\
{ e _ { t } = 2 [ \frac { t } { 2 } ] - 2 , }
\end{array} \quad \left\{\begin{array}{l}
d_{s}=2 s \\
e_{1}=1 \\
e_{t}=2 t-2
\end{array}\right.\right.\right.
$$

for $q=3,5,11$ respectively where $s$ represents any positive integer and $t$ represents any positive integer $\geq 2$.

An example for an even positive $4 \times 4$ matrix with level $q$ and determinant $q^{2}$ is the following:

$$
A=\left(\begin{array}{rrrr}
2 & 1 & \\
1 & 2 & \\
& & 2 & 1 \\
& & 1 & 2
\end{array}\right),\left(\begin{array}{rrrr}
2 & 1 & 0 & 0 \\
1 & 2 & 0 & 1 \\
0 & 0 & 10 & 5 \\
0 & 1 & 5 & 4
\end{array}\right)
$$

for $q=3,5$ respectively.

We may use Siegel's theorem for $m=3$ (resp. 2) if $q=3$ (resp. 5) and instead of (2) we obtain: for $q=3$,

$$
\begin{aligned}
F(\tau, B) & =\frac{252}{13}\left(G_{6}(\tau)-G_{6}(3 \tau)\right)-504 G_{6}(3 \tau) \\
& =1+\frac{252}{13} e^{2 \pi i \tau}+\cdots \cdot
\end{aligned}
$$

where

$$
B=\left(\begin{array}{lll}
A & & \\
& A & \\
& & A
\end{array}\right)^{\prime}
$$

for $q=5$,

$$
\begin{aligned}
F(\tau, B) & =\frac{120}{13}\left(G_{4}(\tau)-G_{4}(5 \tau)\right)+240 G_{4}(5 \tau) \\
& =1+\frac{120}{13} e^{2 \pi i \tau}+\cdots \cdot
\end{aligned}
$$

where $B=\left(\begin{array}{ll}A & \\ & A\end{array}\right) \cdot$ Moreover noticing that for even $m \mathfrak{M}(m)$ is spanned by $\vartheta(\tau, A)^{m}, \vartheta(\tau, M)^{\frac{m}{2}}$ and $\subseteq(m)$ and for odd $m \geq 3 \mathfrak{M}(m)$ is spanned by 
$\vartheta(\tau, A)^{m}, \vartheta(\tau, M)^{\frac{m-1}{2}} \vartheta(\tau, A)$ and $\widetilde{\varsigma}(m)$, the theorem for $q=3,5$ can be proved by induction on $m$ as in the case of $q=2$. For $q=11$, using the fact that all modular forms of degree -2 for $\Gamma_{0}(11)$ is generated by theta functions of degree -2 with level 11, which is proved by Eichler [1] in a more general form, we can prove the theorem only by the dimension formula.

Remark. We proved the theorem for $q=11$ by using a general result of Eichler but we can also prove this like the other case by extending a theorem of Siegel for the case of the even positive quarternary quadratic forms according to a methos of Maads [2].

\section{REFERENCES}

[1] M. Eichler, Über die Darstellbarkeit von Modulformen durch Thetareihen, J. Reine Angew. Math. 195 (1956), 156-171.

[2] H. Maass, Konstruktion ganzer Modulfomen halbzahliger Dimension mit 9 -Multiplikatoren in einer und zwei Variabeln, Abh. Math. Sem. Hamburg, 12, (1938), 133-162.

[ 3 ] B. Schoeneberg, Das Verhalten von mehrfachen Thetareihen bei Modulsubstitutionen, Math. Ann. 116 (1939), 511-523.

[4] C.L. Siegel, Über die analytische Theorei der quadratischen Formen, Ann. of Math. 36 (1935), 527-606.

Mathematical Institute

Nagoya University 